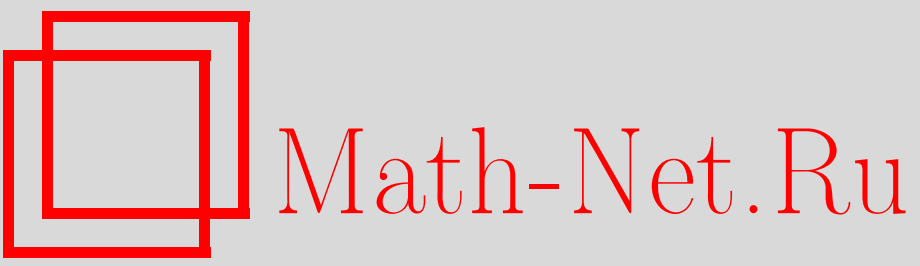

Ф. Ф. Воронов, О. М. Худавердян, Геометрия дифференциальных операторов и нечетные операторы Лапласа, УМH, 2003, том 58, выпуск 1, 179-180

DOI: https://doi.org/10.4213/rm597

Использование Общероссийского математического портала Math-Net.Ru подразумевает, что вы прочитали и согласны с пользовательским соглашением

http://www.mathnet.ru/rus/agreement

Параметры загрузки:

IP: 52.90 .164 .192

26 апреля 2023 г., 13:27:09 


\title{
ГЕОМЕТРИЯ ДИФФЕРЕНЦИАЛЬНЫХ ОПЕРАТОРОВ И НЕЧЕТНЫЕ ОПЕРАТОРЫ ЛАПЛАСА
}

\author{
Ф. Ф. ВоРОнов, О. М. ХУДАВЕРДЯН
}

Пусть $\Delta$ - произвольньй линейный дифференциальньй оператор второго порядка, действующий на функции на (супер)многообразии $M$. В локальных координатах

$$
\Delta=\frac{1}{2} S^{a b} \partial_{b} \partial_{a}+T^{a} \partial_{a}+R .
$$

Главньй символ $\Delta$ есть симметрическое тензорное поле $S^{a b}$, или квадратичная функция $S=$ $\frac{1}{2} S^{a b} p_{b} p_{a}$ на $T^{*} M$. Главный символ может пониматься как симметричная "скобка" на функциgx:

$$
\{f, g\}:=\Delta(f g)-(\Delta f) g-(-1)^{\varepsilon \widetilde{f}} f(\Delta g)+\Delta(1) f g,
$$

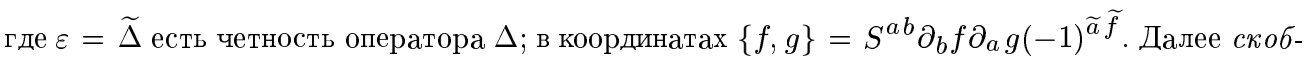
кой в коммутативной алгебре будем назьвать произвольное симметричное бидифференцирование. Задача состоит в описании в геометрических терминах всех операторов $\Delta$ с данным $S^{a b}$, или, что то же самое, порождающих данную скобку $\{f, g\}$. Не уменьшая общности, положим впредь $R=\Delta(1):=0$. Первоначально операторы предполагаются действующими на скалярные функции. По ходу дела у нас естественно появятся пучки операторов, действующих на плотностях произвольного веса. Все рассмотрения применимы как к обычньм многообразиям, так и к супермногообразиям. В суперслучае для нечетных операторов возникают вопросы о тождествах типа Якоби. Задача тесно связана с геометрией формализма Баталина-Вилковыского в квантовой теории поля (описание операторов, порождающих нечетную скобку).

Первое нетривиальное наблюдение состоит в том, что хёрмандеровский субглавный символ $\operatorname{sub} \Delta=\left(\partial_{b} S^{b a}(-1)^{\widetilde{b}(\varepsilon+1)}-2 T^{a}\right) p_{a}$ может быть интерпретирован как "верхняя связность" в расслоении Vol $M$. Точнее, $\gamma^{a}=\partial_{b} S^{b a}(-1)^{\widetilde{b}(\varepsilon+1)}-2 T^{a}$ имеет закон преобразования $\gamma^{a^{\prime}}=$ $\left(\gamma^{a}+S^{a b} \partial_{b} \ln J\right) \frac{\partial x^{a^{\prime}}}{\partial x^{a}}$, где $J=\frac{D x^{\prime}}{D x}$ (якобиан), и задает "контравариантную производную" $\nabla^{a} \rho=\left(S^{a b} \partial_{b}+\gamma^{a}\right) \rho$ на формах объема. Зависящий от системы координат гамильтониан $\gamma=$ $\operatorname{sub} \Delta=\gamma^{a} p_{a}$ играет роль локальной формы связности. Если матрица $S^{a b}$ обратима, то можно опустить индекс $a$ и получить обыкновенную связность. (Подчеркнем, что $\Delta$ действует на функциях, а на многообразии априори нет никакой дополнительной структуры. Pacслоение Vol $M$ и верхняя связность в нем появляются из самого оператора.) Таким образом, $\Delta$ определяется набором данных: скобка на функциях и ассоциированная верхняя связность в Vol $M$.

Определим алгебру плотностей $\mathfrak{V}(M)$ как алгебру формальных линейных комбинаций плотностей произвольных весов $w \in \mathbb{R}$. В $\mathfrak{V}(M)$ есть единица 1 и естественное инвариантное скалярное умножение. Скалярное произведение задается формулой:

$$
\langle\boldsymbol{\psi}, \boldsymbol{\chi}\rangle=\int_{M} \operatorname{Res}\left(t^{-2} \psi(x, t) \chi(x, t)\right) D x .
$$

Мы задаем элементы $\mathfrak{V}(M)$ производящими функциями $\boldsymbol{\psi}(x, t)$, определенными на многообразии $\widehat{M}$. Можно классифицировать дифференцирования алгебры $\mathfrak{V}(M)[1]$. Скобка веса 0 в $\mathfrak{V}(M)$ задается тензором $\left(\widehat{S}^{\widehat{a} \widehat{b}}\right)=\left(\begin{array}{cc}S^{a b} & t \gamma^{a} \\ t \gamma^{a} & t^{2} \theta\end{array}\right)$ на $\widehat{M}$, где $S^{a b}$ задает скобку на $M, \gamma^{a}$ задает ассоциированную с $S^{a b}$ верхнюю связность в $\operatorname{Vol} M$, а член $\theta$ есть геометрический объект второго порядка (зависящий от $S^{a b}$ и $\gamma^{a}$ ), аналогичньй полю Бранса-Дикке в теориях типа Калуцы-Клейна. Набор данных $S^{a b}, \gamma^{a}, \theta$ эквивалентен (неканонически) набору, состоящему из $S^{a b}$, векторного поля и скаляра. Операторы в алгебре $\mathfrak{V}(M)$ записьваются как пучки операторов $\Delta_{w}$, действующих на $w$-плотности. Пучок самосопряжен, если $\left(\Delta_{w}\right)^{*}=\Delta_{1-w}$. Дифференциальный оператор второго порядка в $\mathfrak{V}(M)$ представляется квадратичным пучком $\Delta_{w}=\Delta_{0}+w A+w^{2} B$, где $\Delta_{0}$ есть оператор второго порядка на функциях, а $A$ и $B$ имеют порядки $\leqslant 1$ и 0 . 
Теорема 1. Для алгебры $\mathfrak{V}(M)$ существует взаимно однозначное соответствие между скобками и операторами второго порядка с условием самосопряженности. Скобке с матрицей $\widehat{S}^{\widehat{a} \widehat{b}}$ отвечает порождающий ее “канонический пучок"

$\Delta_{w}=\frac{1}{2}\left(S^{a b} \partial_{b} \partial_{a}+\left(\partial_{b} S^{b a}(-1)^{\widetilde{b}(\varepsilon+1)}+(2 w-1) \gamma^{a}\right) \partial_{a}+w \partial_{a} \gamma^{a}(-1)^{\widetilde{a}(\varepsilon+1)}+w(w-1) \theta\right)$.

Соответствующий оператор в алгебре $\mathfrak{V}(M)$ есть лапласиан, построенньй по $\widehat{S}^{\widehat{a} \widehat{b}}$ и канонической дивергенции на $\widehat{M}[1]$. Через оператор на $w_{0}$-плотностях с данным "неособым" $w_{0} \neq 0, \frac{1}{2}, 1$ проходит единственный канонический пучок. По оператору на функциях пучок восстанавливается с точностью до $w(w-1) f$, где $f$ - скаляр.

Рассмотрим нечетные операторы. Им отвечают нечетные скобки. Заметим, что нечетная симметричная скобка сдвигом четности преврашается в четную антисимметричную. Если $\Delta$ нечетен и $\operatorname{ord} \Delta \leqslant 2$, то ord $\Delta^{2} \leqslant 3$. Условие ord $\Delta^{2} \leqslant 2$ равносильно тождеству Якоби для скобки, порожденной $\Delta$. В этом случае $D=(S,-)$ есть дифференциал в $C^{\infty}\left(T^{*} M\right)$, а для верхней связности $\gamma=\gamma^{a} p_{a}$ имеет смысл понятие кривизны: $F=D \gamma$.

Теорема 2. $\Delta$ есть дифферениирование порожденной им скобки (равносильно оrd $\Delta^{2}$ \$1) тогда и только тогда, когда $D \gamma=0$.

Плоскость $\gamma$ "поглощается" тождеством Якоби для скобки в $\mathfrak{V}(M)$ :

Теорема 3. Тождество Якоби для нечетной скобки в $\mathfrak{V}(M)$ равносильно уравнениям $(S, S)=0,(S, \gamma)=0,(S, \theta)+(\gamma, \gamma)=0,(\gamma, \theta)=0 .\left(\right.$ При этом $\Delta_{w}^{2}=\mathscr{L}_{X}$ для некоторого $X \in \operatorname{Vect}(M)$, автоматически пуассонова.)

СледствиЕ. Если нечетная скобка на $M$, заданная $S^{a b}$, невьрождена, то тождество Якоби для скобки в $\mathfrak{V}(M)$ влечет $\gamma^{a}=S^{a b} \gamma_{b}, \theta=\gamma^{a} \gamma_{a}$, әде $\gamma_{a}=-\partial_{a} \ln \rho, u$ $\rho=e^{\mathscr{A}}$ есть локальная форма обвема. Таким образом, $\Delta$ определяется скобкой на $M$ и “әффективным действием" $\mathscr{A}$.

Условие $\Delta^{2}=0$ в нечетном симплектическом случае дает уравнение Баталина-Вилковыского на действие $\mathscr{A}$. В общем нечетном пуассоновом случае "уравнения Баталина-Вилковыского" пишутся по паре плоских связностей и обладают группоидньм свойством. Они описывают изменение оператора $\Delta^{2}$ на функциях или $\Delta$ на полуплотностях [1], [2].

Теория обобщается для операторов и скобок ненулевого веса. Интересно рассмотреть обобщение на случай операторов высшего порядка (где должны появляться гомотопические алгебры).

\section{СПИСОК ЛИТЕРАТУРЫ}

[1] H. M. Khudaverdian, Th. Voronov. On odd Laplace operators. II; math.DG/0212311. [2] H. M. Khudaverdian, Th. Voronov. On odd Laplace operators // Lett. Math. Phys. 2003 (to appear); math.DG/0205202.

University of Manchester Institute of Science and Technology (UMIST), United Kingdom;

Принято редколлегией Yerevan State University, Armenia

20.11 .2002

E-mail: theodore.voronov@umist.ac.uk, khudian@umist.ac.uk 